\title{
Activating PRKACB somatic mutation in cortisol-producing adenomas
}

\author{
Stéphanie Espiard, ,,2 Matthias J. Knape, ${ }^{3}$ Kerstin Bathon, ${ }^{4}$ Guillaume Assié, ${ }^{1,5}$ Marthe Rizk-Rabin, ${ }^{1}$ \\ Simon Faillot, ${ }^{1}$ Windy Luscap-Rondof, ${ }^{1}$ Daniel Abid, ${ }^{3}$ Laurence Guignat, ${ }^{5}$ Davide Calebiro, ${ }^{4,6,7}$ \\ Friedrich W. Herberg, ${ }^{3}$ Constantine A. Stratakis, ${ }^{2}$ and Jérôme Bertherat ${ }^{1,5}$ \\ ${ }^{1}$ Cochin Institute, Paris Descartes University, CNRS (UMR 8104)/Inserm (U1016), Paris, France. ${ }^{2}$ Section on Endocrinology \\ and Genetics, Eunice Kennedy Shriver National Institute of Child Health and Human Development, NIH, Bethesda, \\ Maryland, USA. ${ }^{3}$ University of Kassel, Department of Biochemistry, Kassel, Germany. ${ }^{4}$ Institute of Pharmacology and \\ Toxicology and Bio-Imaging Center/Rudolf Virchow Center, University of Würzburg, Würzburg, Cermany. ${ }^{5}$ Center for Rare \\ Adrenal Diseases, Endocrinology Department, Cochin Hospital, Assistance Publique Hôpitaux de Paris, Paris, France. \\ ${ }^{6}$ Institute of Metabolism and Systems Research, University of Birmingham, Birmingham, United Kingdom. ${ }^{7}$ Centre of \\ Membrane Proteins and Receptors (COMPARE), Universities of Birmingham and Nottingham, United Kingdom.
}

Mutations in the gene encoding the protein kinase A (PKA) catalytic subunit $\alpha$ have been found to be responsible for cortisol-producing adenomas (CPAs). In this study, we identified by whole-exome sequencing the somatic mutation p.S54L in the PRKACB gene, encoding the catalytic subunit $\beta$ (C $\beta$ ) of PKA, in a CPA from a patient with severe Cushing syndrome. Bioluminescence resonance energy transfer and surface plasmon resonance assays revealed that the mutation hampers formation of type I holoenzymes and that these holoenzymes were highly sensitive to CAMP. PKA activity, measured both in cell lysates and with recombinant proteins, based on phosphorylation of a synthetic substrate, was higher under basal conditions for the mutant enzyme compared with the WT, while maximal activity was lower. These data suggest that at baseline the PRKACB p.S54L mutant drove the adenoma cells to higher CAMP signaling activity, probably contributing to their autonomous growth. Although the role of PRKACB in tumorigenesis has been suggested, we demonstrated for the first time to our knowledge that a PRKACB mutation can lead to an adrenal tumor. Moreover, this observation describes another mechanism of PKA pathway activation in CPAs and highlights the particular role of residue Ser54 for the function of PKA.

Authorship note: SE and MJK contributed equally to this work.

Conflict of interest: The authors have declared that no conflict of interest exists.

Submitted: November 7, 2017 Accepted: March 20, 2018 Published: April 19, 2018

\section{Reference information:} JCI Insight. 2018;3(8):e98296. https://doi.org/10.1172/jici. insight.98296.

\section{Introduction}

The cAMP pathway is one of the most important signaling pathways in adrenocortical cells, and is involved in their regulation of growth and steroidogenesis. Under normal conditions, adrenocorticotropic hormone (ACTH) binds to its G protein-coupled receptor, the melanocortin 2 receptor, which via the activation of the $\alpha$-stimulating subunit of the guanine nucleotide-binding protein $(\mathrm{Gs} \alpha)$, stimulates adenyl cyclase and thereby cAMP production. Protein kinase A (PKA) is a tetrameric enzyme composed of a regulatory (R) dimer and 2 catalytic (C) subunits. Binding of 2 cAMP molecules to each R subunit leads to holoenzyme dissociation and subsequent phosphorylation of substrates by the $\mathrm{C}$ subunits (1). There are 4 different $\mathrm{R}$ subunits $(\mathrm{RI} \alpha$, $\mathrm{RI} \beta, \mathrm{RII} \alpha$, and $\mathrm{RII} \beta)$ and $4 \mathrm{C}$ subunits $(\mathrm{C} \alpha, \mathrm{C} \beta, \mathrm{C} \gamma$, and protein kinase $\mathrm{X}) . \mathrm{C} \alpha$ and protein kinase $\mathrm{X}$ are ubiquitously expressed, whereas $C \beta$ has a more limited pattern of expression and $C \gamma$ is mainly expressed in testis (2). Type I PKA holoenzyme corresponds to the tetramers formed with the type I R subunits (RI $\alpha$ and RI $\beta$ ), while type II PKA holoenzyme corresponds to the tetramers formed with type II R subunits (RII $\alpha$ and RII $\beta$ )

Activation of the PKA pathway has been described in several adrenal diseases with Cushing syndrome (CS): germline mutations of protein kinase cAMP-dependent type I regulatory subunit $\alpha$ (PRKAR1A encoding the subunit RI $\alpha$ of PKA) cause Carney complex and primary pigmented nodular adrenal disease; mosaic mutations of GNAS (encoding for Gs $\alpha$ ) lead to McCune-Albright syndrome, which can be associated with bilateral adrenal hyperplasia; germline variants of the gene encoding for phosphodiesterase $11 \mathrm{~A}$ favor the development of primary pigmented nodular adrenal disease and primary bilateral macronodular adrenal hyperplasia, and germline and somatic mutations of the gene encoding for phosphodiesterase $8 \mathrm{~B}$ have been described in micronodular adrenocortical disease and primary bilateral macronodular adrenal 
A

leukocytes

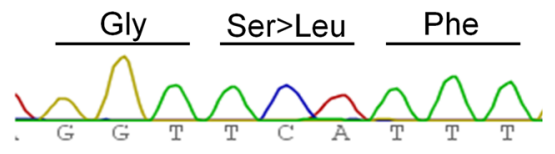

tumor

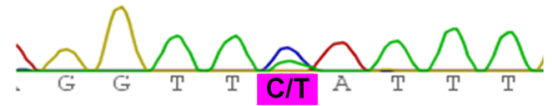

B

\section{Interspecies}

Zebrafish

Chicken

Xenopus

Human

Mouse

Gorilla

Sheep

Human

PRKACB

PRKACA
RKWESPQQSTTCLDDFDRQKTLGTGSFGRVLLVKHKASDQYYAMKVLDKQKVV RKWESPPQNTAGLDDFERQKTLGTGSFGRVMMVKHKATEQYYAMKILDKQKVV RKWETPPQNTASLDDFERIKTLGTGSFGRVMLVKHKGAEQYYAMKILDKQKVV KKWENPTQNNAGLEDFERKKTLGTGSFGRVMLVKHKATEQYYAMKILDKQKVV RKWENPPPSNAGLEDFERKKTLGTGSFGRVMLVKHKATEQYYAMKILDKQKVV KKWENPTQNNAGLEDFERKKTLGTGSFGRVMLVKHKATEQYYAMKILDKQKVV KKWENPAPNNAGLEDFERKKTLGTGSFGRVMLVKHKATEQYYAMKILDKQKVV

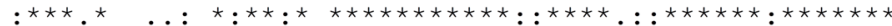

KKWENPTQNNAGLEDFERKKTLGTGSFGRVMLVKHKATEQYYAMKILDKQKVV KKWESPAQNTAHLDQFERIKTLGTGSFGRVMLVKHKETGNHYAMKILDKQKVV

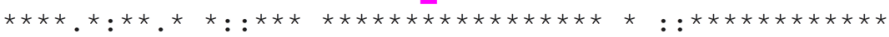

Figure 1. Somatic mutation at the conserved serine 54 of PRKACB. (A) Partial electropherograms of the $P R K A C B$ gene showing a canonical sequence in the leukocytes of the patient and the c.161C>T (S54L) mutation in the tumor (according to the variant NM_002731). (B) Multiple alignments of PRKACB sequences (performed with CLUSTAL 0 1.2.1) showing that the residue $\mathrm{S} 54 \mathrm{~L}$ is conserved between different isoforms and species. (C) Crystal structure of the PKA holoenzyme complex. The catalytic subunit $(C \alpha)$ is in white, and the regulatory subunit $(\mathrm{R} \mid \alpha)$ is in blue. On the left, magnification of the active site shows Ser54 of the glycine-rich loop, which is involved in binding of the cosubstrate ATP (orange sticks) and substrates (here the pseudosubstrate sequence of $\mathrm{RI} \alpha$ ). S54L is located in the immediate vicinity of the inhibitor sequence of the regulatory subunit. The structure (PDB 2QCS) was visualized using PyMOL v1.3 (Schrödinger LLC).
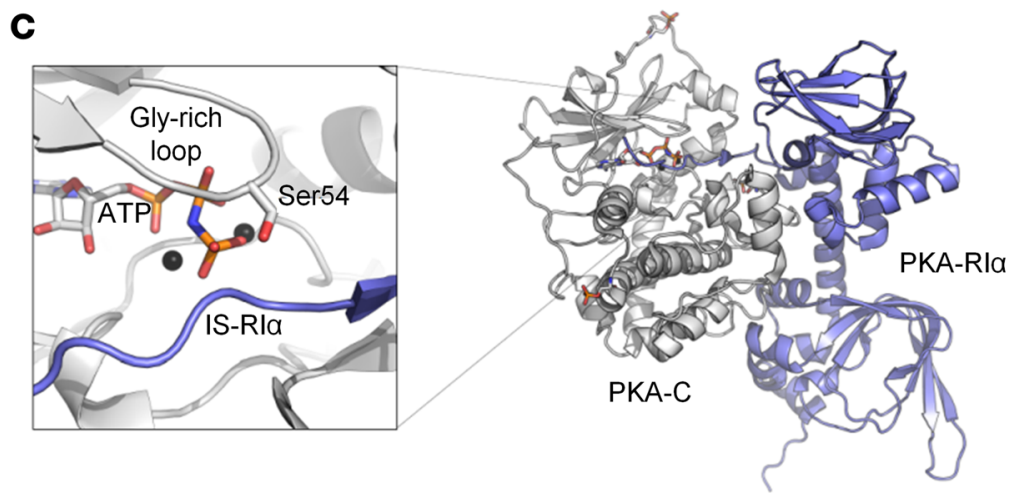

hyperplasia, respectively (3). Somatic mutations of PRKAR1A and GNAS have been found in cortisol-producing adenomas (CPAs) (3). In 2014, several groups demonstrated the involvement of somatic mutations of protein kinase cAMP-activated catalytic subunit $\alpha$ (PRKACA, encoding for the C $\alpha$ subunit of PKA) in about $40 \%$ of CPAs (4-7). All PRKACA mutations found in CPA to date lead to loss of interaction with the $\mathrm{R}$ subunits and consequently an increase of total PKA activity (8).

In this study, we identified a mutation in the protein kinase cAMP-activated catalytic subunit $\beta$ gene (PRKACB, encoding the $\mathrm{C} \beta$ isoform of $\mathrm{PKA}$ ) and confirmed in vitro that the mutation affects type I PKA holoenzyme function.

\section{Results}

Identification of a potentially novel PRKACB gene mutation. To identify new candidate genes responsible for $\mathrm{CPA}$, we performed whole-exome sequencing of 6 paired leukocyte and tumor DNA samples. Somatic mutations in genes involved in the cAMP/PKA pathway were found in 5 of 6 tumors including missense mutations in PDE8B $(n=1)$, GNAS $(n=2), \operatorname{PRKACA}(n=1)$, and PRKACB $(n=1)$. Other mutations identified after filtering are presented in Supplemental Table 1 (supplemental material available online with this article; https://doi.org/10.1172/jci.insight.98296DS1). In the adenoma harboring the PRKACB mutation, no other relevant genetic defects could be identified. Sanger sequencing of tumor and leukocyte DNA of the patient confirmed the presence of the somatic mutation c.161C>T, p.S54L in PRKACB (NM_002731) (Figure 1A). Sequencing of coding sequences and flanking introns of $P R K A C B$ in the tumor DNA from another 21 WT-PRKACA CPAs did not show any other mutations. In addition, reviewing the literature indicated that no other PRKACB mutation was described in published whole-exome sequences of 164 CPAs $(4-7,9)$.

In silico modeling of S54L mutant. Serine 54 (Ser54) is conserved in the human $\mathrm{C} \alpha, \mathrm{C} \beta$, and $\mathrm{C} \gamma$ sequences, as well as in that of other species (Figure 1B). This residue is located at the tip of the glycine-rich loop 
A

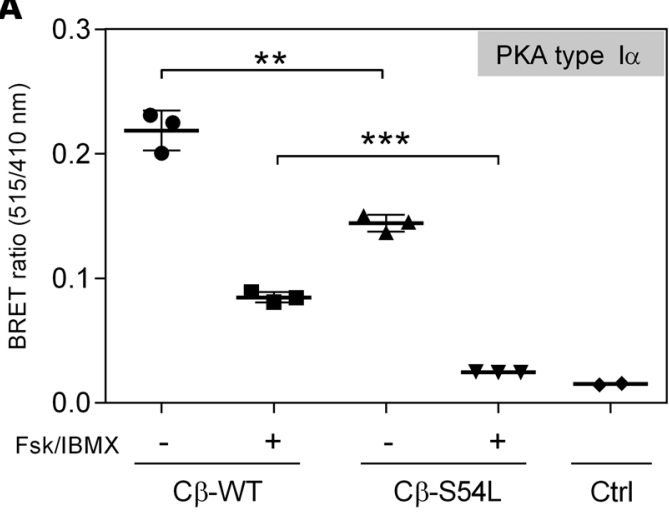

B

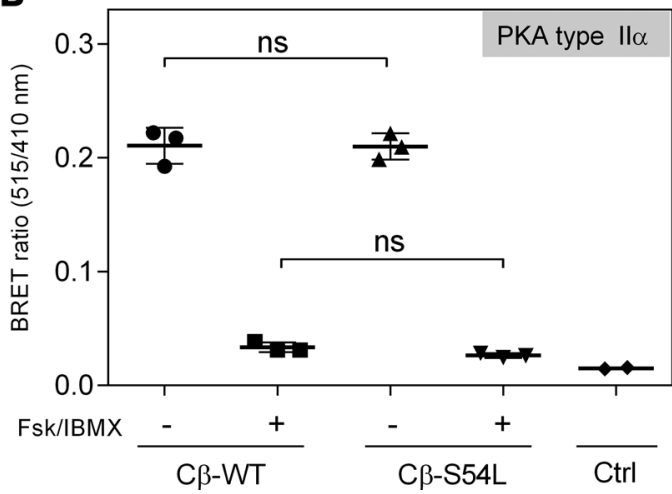

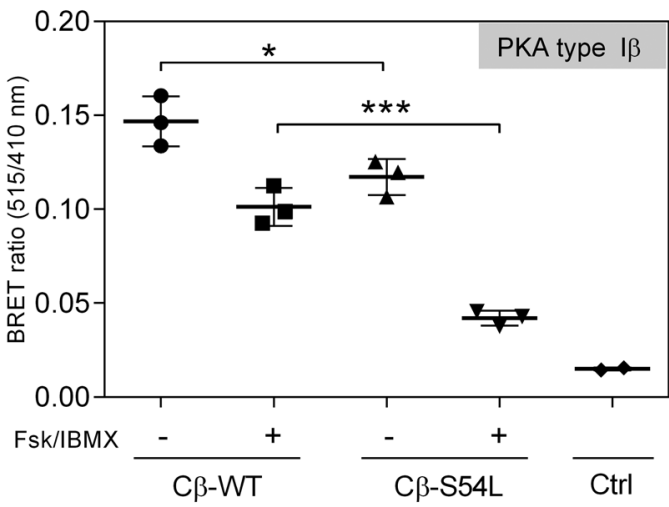

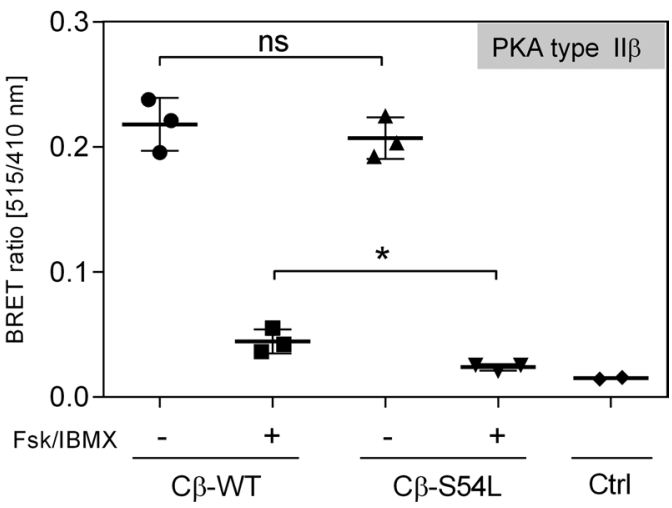

Figure 2. Mutant type I PKA holoenzyme stability is decreased in HEK293 cells. Bioluminescence resonance energy transfer (BRET) experiments were used to test PKA type I (A) and type II (B) holoenzyme formation and dissociation. HEK293 cells were cotransfected with RLuc-tagged regulatory subunits (RI $\alpha$ or RI $\beta$ in panel A and RIl $\alpha$ or RII $\beta$ in panel B) as a donor (emission $410 \mathrm{~nm}$ ) and GFP-tagged catalytic subunits (wild-type [WT] or mutant [S54L] C $\beta$ isoform 1) as an acceptor (emission $515 \mathrm{~nm}$ ). Cells were treated either with buffer (basal condition, black boxes) or with $50 \mu \mathrm{M}$ adenylyl cyclase activator forskolin (Fsk) and $100 \mu \mathrm{M}$ phosphodiesterase inhibitor 3-isobutyl-1-methylxanthine (IBMX) to induce holoenzyme dissociation (stimulated condition, white boxes). Transfection of the empty RLuc8 vector was used as control (Ctrl). (A) The BRET ratio is significantly lower in basal and stimulated conditions for type I holoenzymes with the S54L mutant compared with the WT, indicating a decrease in mutant holoenzyme formation and stability. (B) No significant differences in BRET ratio were observed under basal conditions for the type II holoenzymes, indicating that the S54L mutation does not disturb the holoenzyme formed with type II regulatory subunits. Data from 3-6 replicates from 3 independent experiments are represented as dot plots and analyzed by unpaired $t$ test with Sidak's post hoc test for multiple comparisons. ${ }^{*} P<0.05$; ${ }^{* *} P<0.01$; ${ }^{* *} P<0.001$. ns, not significant.

(G-loop) (Figure 1C). The mutation S54L was predicted to be damaging by MutationTaster (probability: 0.99999) and SIFT (score: 0), and benign by Polyphen2 (score: 0.441).

Patient description. The patient was a 41-year-old woman referred to our clinic for the investigation and treatment of CS. She developed all the stigmata of clinical CS complicated by hypertension, venous thrombosis, osteoporosis with vertebral fractures, dyslipidemia, and hypertrophic obstructive cardiomyopathy. Biological evaluation showed ACTH-independent CS: loss of cortisol circadian rhythm with plasma midnight cortisol at $217 \mathrm{ng} / \mathrm{ml}$ (normal [N]: $<75 \mathrm{ng} / \mathrm{ml}$ ), midnight salivary cortisol at $6.3 \mathrm{ng} / \mathrm{ml}(\mathrm{N}:<2 \mathrm{ng} / \mathrm{ml})$, urinary free cortisol (UFC) at $333 \mu \mathrm{g} / 24$ hours (N: 25-90), and lack of cortisol suppression upon standard dexamethasone suppression test $(2 \mathrm{mg} /$ day) (UFC at $451 \mu \mathrm{g} / 24$ hours $[\mathrm{N}:<10])$. There was no aldosterone or androgen oversecretion. Abdominal computed tomography (CT) showed a 3-cm left-adrenal nodule with a spontaneous density of 47 Hounsfield units. On magnetic resonance imagery, the left nodules did not demonstrate a loss of signal intensity on opposed-phase images and the drop in signal intensity was lower than $20 \%$ in out-of-phase images in favor of an atypical adenoma. ${ }^{18} \mathrm{~F}$-fluorodeoxyglucose positron emission tomography-CT and ${ }^{131} \mathrm{I}$-noriodocholesterol scintigraphy showed increased uptake by the left nodules. The patient underwent left adrenalectomy. Histological examination showed a 3-cm yellow and pigmented adenoma compound of compact adrenocortical cells with islet of spongiocytes cells and clusters of adipocytes with lymphoid infiltration. The Weiss score was 1 . The patient was cured of CS and developed adrenal insufficiency postoperatively, for which she was treated.

PRKACB mutation S54L affects stability for type I but not for type II PKA holoenzyme. To provide in vitro evidence for the pathogenicity of the S54L mutation, we first tested whether the S54L mutation affects PKA 
A

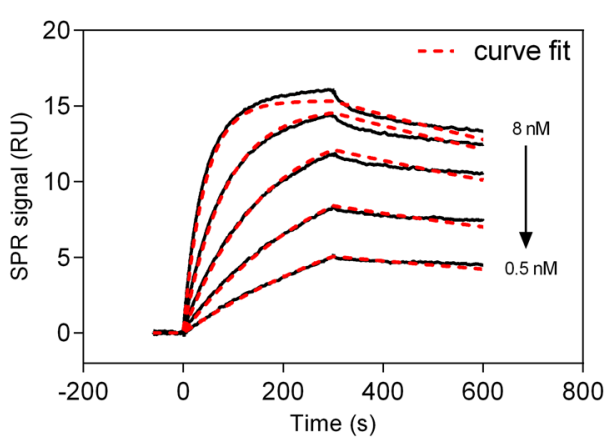

C

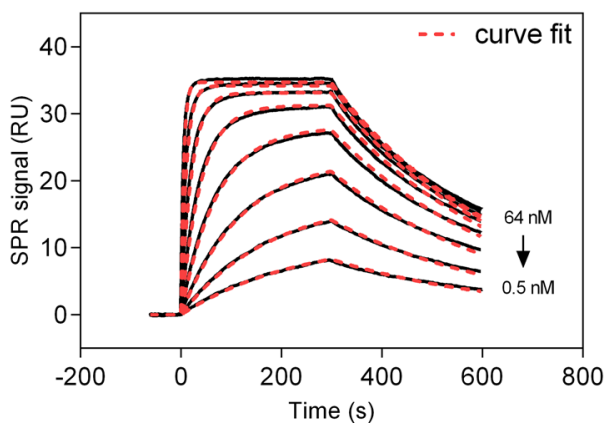

B

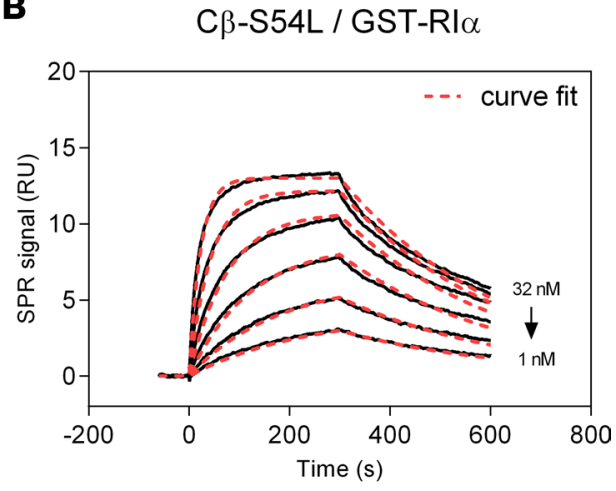

D

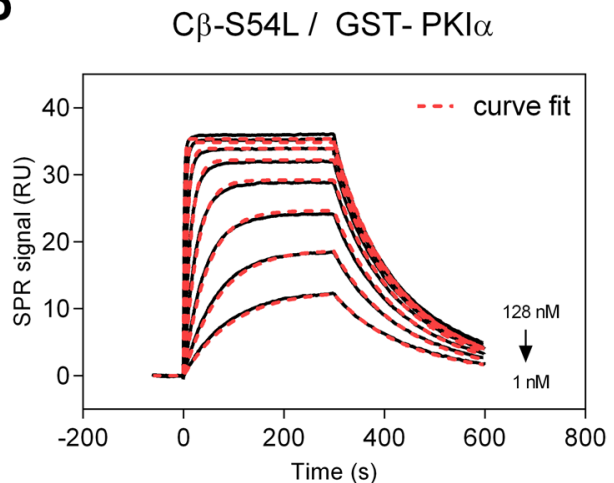

Figure 3. Binding of PKA-C $\beta$ to physiological pseudosubstrate inhibitors. Binding affinity of $C \beta-W T(A$ and $\mathbf{C})$ or $C \beta-S 54 L$ (B and $\mathbf{D})$ was determined using surface plasmon resonance (SPR). For this, recombinant Rl $\alpha$ subunit (A and $\mathbf{B}$ ) or the protein kinase inhibitor $\alpha$ (PKI $\alpha$ ) (C and $\mathbf{D})$ were captured on a sensor chip. Recombinant C $\beta-W T$ ( $\mathbf{A}$ and $\mathbf{C}$ ) or C $\beta$-S54L (B and $\mathbf{D}$ ) were injected for 300 seconds at different concentrations (association). Dissociation of the complex was monitored for 300 seconds. Equilibrium binding constants and rate constants were determined using a Langmuir 1:1 binding model (curve fits are depicted as red dashed lines in representative plots) and are summarized in Tables 1 and 2 . The reduced affinities for RI $\alpha$ and PKI $\alpha$ determined for the mutant $\mathrm{C} \beta$-S54L compared with the WT protein are mainly due to faster complex dissociation (see Tables 1 and 2 for dissociation rates). RU, response units.

holoenzyme formation. We used a cell-based bioluminescence resonance energy transfer (BRET) assay as described previously (10) based on transient coexpression of a luciferase-tagged R subunit (either RI $\alpha$, RI $\beta$, RII $\alpha$, or RII $\beta$ ) as a donor and GFP-tagged versions of either the WT or mutant (S54L) C $\beta$ subunit (isoform 1, C $\beta 1$ ) as an acceptor. The BRET ratio (ratio of acceptor and donor emission) was significantly lower under basal conditions as well as after stimulation with forskolin and 3-isobutyl-1-methylxanthine (IBMX) for type I mutant holoenzymes (RI $\alpha / C \beta$-S54L and RI $\beta / C \beta$-S54L) compared with the respective WT holoenzymes (Figure 2A). Formation of type II holoenzymes (RII $\alpha / C \beta$-S54L and RII $\beta / C \beta$-S54L) was not affected by the S54L mutation (Figure $2 \mathrm{~B}$ ). In order to validate that the S54L mutation affects type I holoenzyme formation, we expressed mutant and WT C $\beta 1$ as recombinant proteins in E. coli and checked for holoenzyme formation in vitro using surface plasmon resonance (SPR). Compared with WT, the S54L mutant showed a 10-fold-reduced affinity for the RI $\alpha$ subunit (Figure 3, A and B, and Table 1). This was mainly due to a 4 -fold-faster dissociation rate reflecting weaker interaction of the holoenzyme

Table 1. Rate and dissociation constants for the interaction of RI $\alpha$ with C $\beta$-WT or C $\beta-S 54 L$

\begin{tabular}{lcccc}
\hline & $\boldsymbol{k}_{\text {ass }}[\mathbf{1} / \mathrm{Ms}]$ & $\boldsymbol{k}_{\text {diss }}[\mathbf{1} / \mathbf{s}]$ & $\boldsymbol{K}_{\mathbf{D}}[\mathrm{nM}]$ & $\boldsymbol{N}$ \\
C $\beta$-WT and Rl $\alpha$ & $3.4( \pm 0.6) \times 10^{6}$ & $5.3( \pm 0.9) \times 10^{-4}$ & $0.2( \pm 0.05)$ & 3 \\
C $\beta$-S54L and Rl $\alpha$ & $1.2( \pm 0.1) \times 10^{6}$ & $2.2( \pm 0.8) \times 10^{-3}$ & $1.8( \pm 0.5)$
\end{tabular}

Values are the mean $\pm \mathrm{SD}$. $k_{\text {ass }}$, association rate constant; $k_{\text {diss }}$, dissociation rate constant; $K_{D^{\prime}}$, equilibrium dissociation constant; $N$, number of independent experiments (each with a separate protein preparation). 
Table 2. Rate and dissociation constants for the interaction of PKI with C $\beta$-WT or C $\beta-S 54 L$

\begin{tabular}{lcccc}
\hline & $\boldsymbol{k}_{\text {ass }}[\mathbf{1} / \mathrm{Ms}]$ & $\boldsymbol{k}_{\text {diss }}[\mathbf{1 / s}]$ & $\boldsymbol{K}_{\mathbf{D}}[\mathrm{nM}]$ & $\boldsymbol{N}$ \\
C $\beta$-WT and PKI $\alpha$ & $3.9( \pm 0.9) \times 10^{6}$ & $2.1( \pm 0.7) \times 10^{-3}$ & $0.6( \pm 0.4)$ & 3 \\
C $\beta$-S54L and PKI $\alpha$ & $5.8( \pm 0.9) \times 10^{6}$ & $9.0( \pm 0.7) \times 10^{-3}$ & $1.6( \pm 0.2)$ & 3
\end{tabular}

Values are the mean $\pm \mathrm{SD}$. $k_{\text {ass' }}$ association rate constant; $k_{\text {diss }}$, dissociation rate constant; $K_{D}$, equilibrium dissociation constant; $N$, number of independent experiments (each with a separate protein preparation).

complex (Table 1). Injecting $30 \mathrm{nM}$ cAMP after holoenzyme formation resulted in pronounced dissociation of the mutant holoenzyme, while the WT was almost unaffected at this concentration (Figure 4A). Interestingly, inhibition by the other physiological inhibitor of PKA, heat-stable protein kinase inhibitor $\alpha$ $(\mathrm{PKI} \alpha)$, was only slightly affected (Figure 3, C and D, and Table 2).

The increased cAMP sensitivity of the S54L type I holoenzyme was verified in a spectrophotometric kinase activity assay. The activation constant $\left(K_{\text {act }}\right)$, reflecting the concentration of cAMP required to obtain $50 \%$ of maximal activity, was 3-fold higher for the WT holoenzyme than for the mutant (Table 3 ). In order to verify this higher cAMP sensitivity, we again utilized BRET to investigate the time course of holoenzyme activation in living cells. After injection of a cell-permeant cAMP analog (8-Br-cAMP-AM), cells expressing the WT holoenzyme showed slow and only partial dissociation of the holoenzyme, while those expressing the mutant holoenzyme showed rapid and complete dissociation (Figure 4B).

PRKACB mutation S54L increases basal PKA activity. We then examined if this reduced holoenzyme stability observed with the mutant C $\beta$-S54L leads to higher basal PKA activity. For this, we coexpressed C $\beta$ (Flagtagged version of isoform 1) and RI $\alpha$ in HEK293 cells and checked the lysates for phosphotransferase activity. PKA activity was measured based on the phosphorylation of the synthetic substrate kemptide (LRRASLG) in cell lysates. Indeed, the basal PKA activity of C $\beta$-S54L was higher compared with WT (Figure 5A). However, after stimulation with cAMP, the activity of the mutant enzyme was lower. The lower phosphotransferase activity observed for the mutant was verified using purified recombinant PKA C subunits produced in E. coli. The C $\beta$-S54L mutant showed an almost 3-fold-reduced specific activity using kemptide as a substrate (Figure 5B). It has to be kept in mind that the specific activity observed for C $\beta 1$-WT with more than $40 \mathrm{U} / \mathrm{mg}$ is more than twice as high as the activity determined for isoform 1 of $\mathrm{C} \alpha(\mathrm{C} \alpha 1)$. Thus, with about $16 \mathrm{U} / \mathrm{mg}$ the S54L mutant displays almost the same specific activity as the major isoform $\mathrm{C} \alpha 1$.

Immunostaining of PRKACB. Immunostaining of $C \beta$ on paraffin tumor sections of the CPA carrying the S54L PRKACB mutation showed an increased expression of $C \beta$ in the nucleus compared with other adenomas, suggesting an activation of the PKA pathway in this tumor (Figure 6).

\section{Discussion}

Somatic mutations of PRKACA cause about $40 \%$ of CPAs, while mutations of the PRKAR1A and GNAS genes have been described more rarely. Here we describe what we believe to be a new mechanism of activation of the PKA pathway by a somatic activating mutation of $P R K A C B$ in a CPA responsible for a severe CS. The S54L PRKACB mutation leads to increased basal PKA activity in adrenocortical cells, as is the case with GNAS and PRKACA mutations $(4,9)$.

The particular function of Ser54 has been previously investigated (11). This residue is located at the tip of the G-loop, which is part of the small amino-terminal lobe of the C subunit. The G-loop is a conserved

\section{Table 3. Activation constants of the WT and mutant type I $\alpha$ PKA holoenzyme}

\begin{tabular}{lcc}
\hline & $\boldsymbol{K}_{\text {act }}$ [nM] & $\boldsymbol{N}$ \\
C $\beta$-WT and RI $\alpha$ & $31.8 \pm 8.3$ & 5 \\
C $\beta$-S54L and RI $\alpha$ & $9.4 \pm 2.2$ & 2
\end{tabular}

Values are the mean \pm SD. $K_{a c t}$, activation constant; $N$, number of independent protein preparations (each experiment was measured at least in duplicate). feature of all protein kinases and is critical for the binding of the cosubstrate ATP (adenosine triphosphate) and substrates $(11,12)$. Closing of the active site is associated with the movement of the G-loop and therefore necessary for stable substrate or inhibitor binding. Biochemical studies of Ser54 in C $\alpha$ showed that the removal of its side chain (S54G mutant) did not result in alteration of kinase activity and sensitivity to $\mathrm{R}$ subunits (11). On the contrary, substitution of the serine with a proline led to a decrease of the phosphotransfer ability of the enzyme 
A
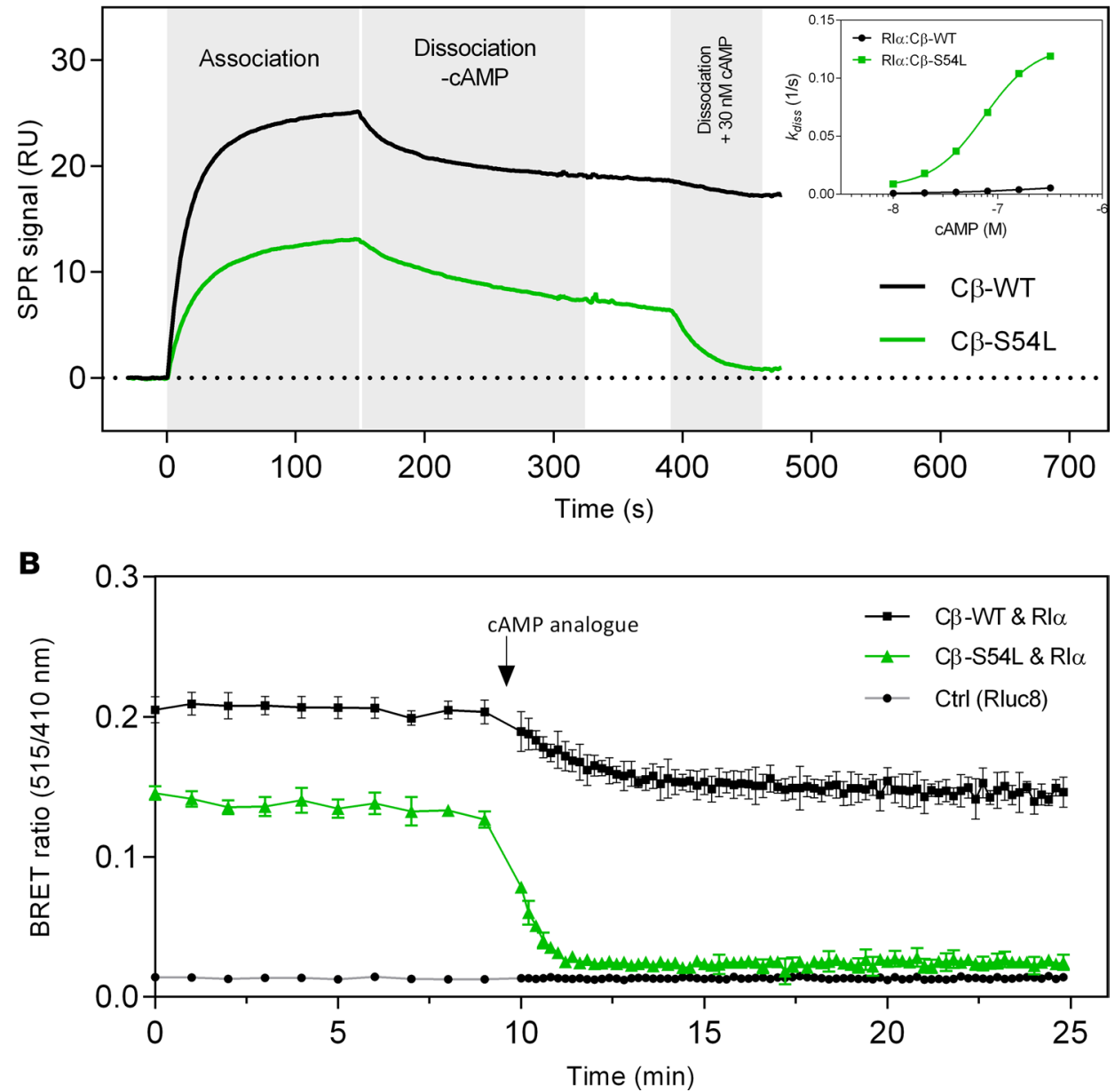

Figure 4. Mutant type I PKA holoenzyme is more sensitive to cAMP. (A) In this surface plasmon resonance (SPR) experiment, recombinant Rl $\alpha$ subunit was captured on a sensor chip and WT or S54L C $\beta$ were injected for 150 seconds (15 nM concentration in this representative experiment), demonstrating a decrease of the mutant holoenzyme complex formation. After 150 seconds the system was switched to buffer, allowing the monitoring of complex stability in the absence of cAMP. After 400 seconds, supplementation of buffer with $30 \mathrm{nM}$ cAMP caused a rapid dissociation of the mutant holoenzyme, whereas the WT holoenzyme was almost unaffected. The inset shows the dissociation rate at several cAMP concentrations. These data clearly demonstrate an increased cAMP sensitivity of the mutant type I holoenzyme. (B) In this bioluminescence resonance energy transfer (BRET) time-course experiment, $100 \mu \mathrm{M}$ cell-permeant CAMP analog (8-Br-CAMP-AM) was injected after a 10-minute baseline measurement. A rapid and full dissociation was observed for the mutant holoenzyme, while the WT showed slow and only partial dissociation. Values are given as mean \pm SD from 6 individual transfections. RU, response units.

and to higher $K_{m}$ values for ATP and the peptide substrate kemptide. In addition, the S54P mutant exhibited a strongly reduced sensitivity to inhibition by type I R subunits (11). Leucine and proline are both amino acids with aliphatic side chains. It is also likely that the S54L mutation restricts the correct positioning of the G-loop. In contrast to serine, the bulky aliphatic side chain of leucine is sterically constrained and will likely hamper substrate (probably contributing to the lower maximal PKA activity) as well as pseudosubstrate binding.

The majority of studies on PKA function have been performed with $C \alpha 1$. Human $C \alpha 1$ and $C \beta 1$ isoforms harbor $91 \%$ homology in their amino acid sequence (2). In vitro, their biochemical properties differ in the affinities for certain peptide substrates and the absence of inhibition of C $\beta 1$ activity for high concentrations of substrates (13). C $\beta$-knockout mouse models suggest distinct roles for $C \beta$ and $C \alpha$ in neuronal development and hippocampal plasticity $(14,15)$. An involvement of $\mathrm{C} \beta$ in cancer has been demonstrated, with studies indicating that $C \beta$ is playing either a role as a tumor suppressor gene (16) or as an oncogene $(17,18)$, depending on the cell model tested. It has been suggested that upregulation of $P R K A C B$ is critical for c-MYC-associated tumorigenesis $(17,19)$. Specific functions of C $\beta$ versus $C \alpha$ in the adrenal cortex, where $C \beta$ is also expressed, have not been studied (20).

Constitutive activation of the PKA pathway leads to tumor development and endocrine overfunction, as observed in toxic thyroid adenoma and growth hormone-producing pituitary adenoma (21). 
A

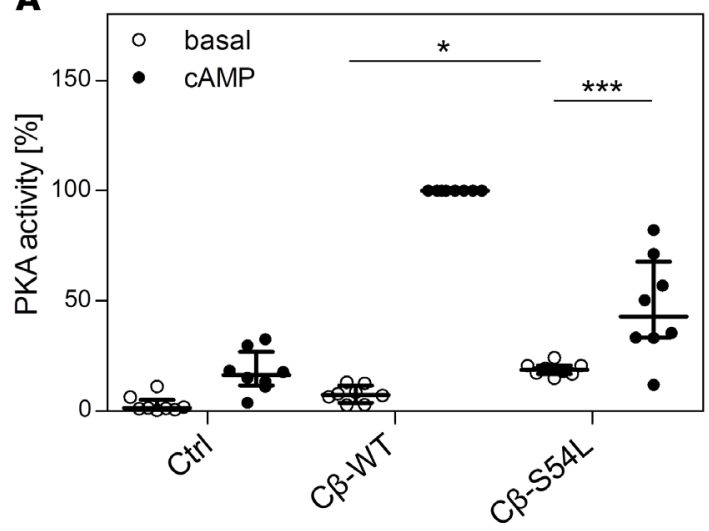

B

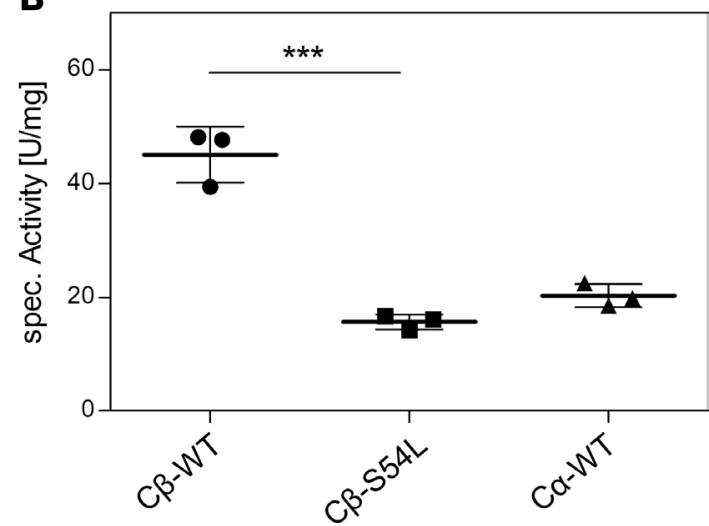

Figure 5. The mutation S54L causes increased phosphotransferase activity under basal conditions. PKA activity was determined by quantifying the phosphorylation of a synthetic fluorescently labeled peptide (kemptide) in HEK293 cell lysates (A) or with unlabeled kemptide for purified recombinant catalytic subunits (B). In detail, (A) PKA activity was tested in lysates of HEK293 cells cotransfected with a combination of Rl $\alpha$ and either WT or S54L C $\beta 1$. Under basal conditions (no CAMP stimulation), the mutant holoenzyme showed higher PKA activity than the WT. In the presence of cAMP, maximal activity was lower for the mutant enzyme. Transfection of the empty pcDNA vector was used as control (Ctrl). Data from 4 independent experiments are represented as dot plots and were analyzed by ANOVA with Sidak's post hoc test for multiple comparisons. (B) PKA activity of purified recombinant Rl $\alpha$ and catalytic subunits ( $C \beta-\mathrm{WT}, \mathrm{C} \beta-\mathrm{S} 54 \mathrm{~L}$, and $\mathrm{C} \alpha-\mathrm{WT})$ revealed an almost 3-fold-reduced phosphotransferase activity $(15.6 \pm 1.3 \mathrm{U} / \mathrm{mg})$ for $C \beta$ - $554 \mathrm{~L}$ compared with $C \beta$-WT ( 45.1 $\pm 4.9 \mathrm{U} / \mathrm{mg})$. The specific activity of the major isoform $C \alpha 1$ was determined as a control $(20.3 \pm 2.0 \mathrm{U} / \mathrm{mg})$. Data from 3 independent protein preparations are represented as dot plots and were analyzed by unpaired $t$ test with Sidak's post hoc test for multiple comparisons. ${ }^{*} P<0.05 ;{ }^{* *} P<0.001$.

CPA is the most frequent cause of ACTH-independent CS. Beyond the typical clinical picture, overt CS is associated with severe comorbidities and increased mortality $(22,23)$. Patients whose tumors harbor PRKACA mutations present at a younger age at diagnosis, higher cortisol levels, and, interestingly, smaller-size tumors than CPAs without mutations $(24,25)$. The present patient had as well an overt CS and a young age at diagnosis, but the size of the adenoma seems to be larger than described for $P R K A C A$ mutation (25). This could be consistent with an additional oncogenic function described for $P R K A C B$. However, with a Weiss score of 1 the patient's adenoma had no characteristics of malignancy. The Cancer Genome Atlas reveals alteration of $P R K A C B$ in various cancer (26) but not in adrenocortical carcinoma (27).

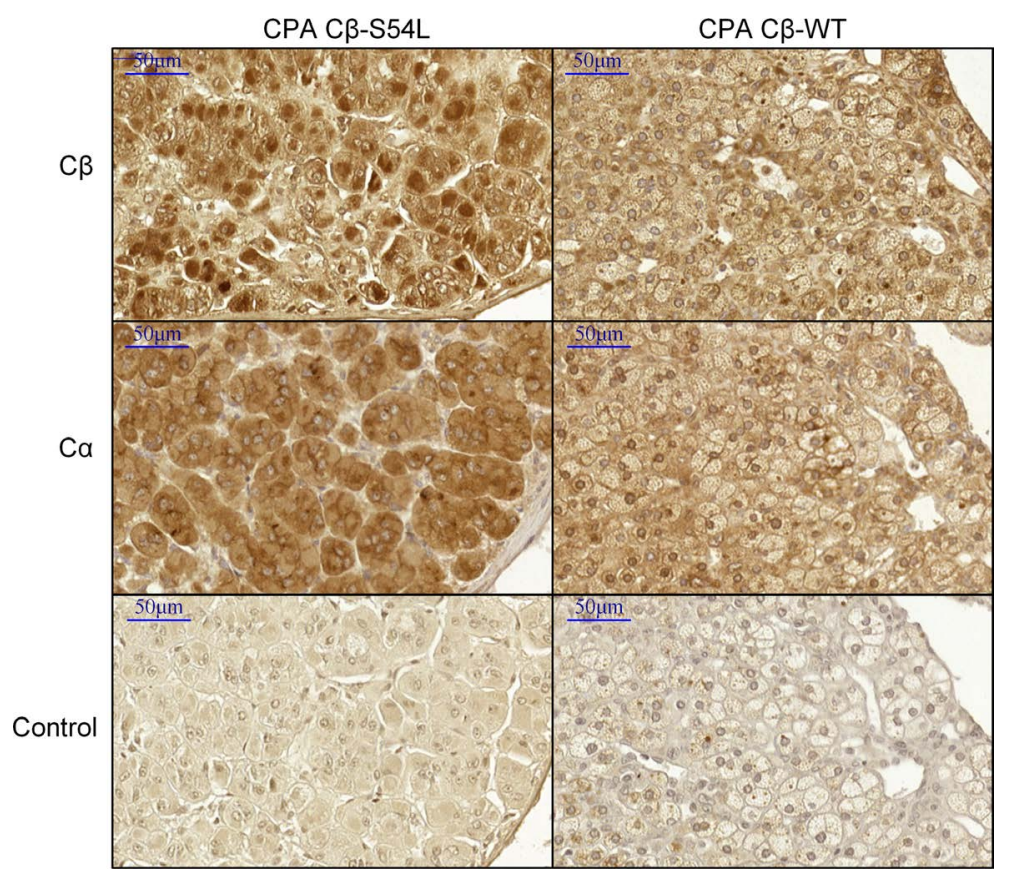

Figure 6. C $\beta$ is localized in the nucleus in the $C \beta-S 54 L$ adenoma. Immunohistochemistry for $C \alpha$ and $C \beta$ in the $\mathrm{S} 54 \mathrm{~L}$-mutated cortisol producing adenoma (CPA) and in a representative WT CPA $(n=2)$. A reinforcement of staining with regard to the nucleus is observed in the S54L-mutated adenoma compared with faint membrane (cytoplasmic and perinuclear) staining in the WT adenoma, whereas $C \alpha$ localization was cytoplasmic in both adenomas. This nuclear localization of $C \beta$ in the mutant adenoma suggests an activation of C $\beta-S 54 \mathrm{~L}$. Scale bars: $50 \mu \mathrm{m}$. 
Recently, a triplication of $P R K A C B$ has been described in a patient with Carney complex who presented with a somatotroph adenoma, pigmented spots, and skin myxomas. This 19-year-old patient had not presented with adrenal disease at the time of publication. A mouse model, overexpressing the $P R K A C B$ gene, showed an increase of growth hormone levels at a young age (28). Thus, $P R K A C B$ may be considered as a candidate gene for tumors associated with endocrine hyperfunction. In sporadic somatotroph adenomas, sequencing of the $P R K A C B$ gene did not show any mutations but sequencing was limited to the region around codon 206 where the mutation hotspot of PRKACA in CPA is located (29).

In conclusion, $P R K A C B$ mutations lead to adrenal CS by the constitutive activation of the PKA pathway. This observation is the first demonstration to our knowledge of the involvement of a PRKACB sequence alteration in tumors. The data presented suggest that the $C \beta$ subunit may have specific functions in the adrenal cortex. For the PKA research field, this study highlights the importance of the serine (Ser54) at the tip of the G-loop for kinase regulation and function.

\section{Methods}

Study approval. The study was approved by the Institutional Review Board of Paris Ile de France III and patients' written informed consent was obtained.

Genetic testing. A total of 6 patients who had undergone surgery for CPA were included in the study. The tumor samples were obtained prospectively by the Cortico-medullo adrenal Tumors Endocrine Network tumor bank (30). DNA was extracted from fresh-frozen tissues and checked for signs of degradation as previously described (31). Exome DNA was captured using the SureSelectXT Human All Exon version 4 Kit (Agilent), following the manufacturer's protocol, and then sequenced on a pair of SOLiD 5500x flowchips (Supplemental Methods). The PRKACB coding and the flanking intronic sequences were amplified by PCR both in tumor DNA from 21 patients presenting with CPA. Both strands of the amplified products were directly sequenced with forward and reverse primers (Supplemental Table 2).

In silico modeling. Somatic variants were evaluated by MutationTaster (http://www.mutationtaster. org/) (32), Polymorphism Phenotyping v2 algorithm tool (PolyPhen-2) (http://genetics.bwh-.harvard. edu/pph2) (33), and the SIFT (Sorting Tolerant From Intolerant) algorithm (http://sift.jcvi.org) (34) to predict the possible impact of the amino acid substitution on the structure and function of a human protein

Patients. The clinical data of the patient harboring the PRKACB mutations were collected retrospectively.

$D N A$ constructs and cell culture. The PRKACB sequence WT (NM_002731) and mutant were introduced into different expression vectors according to established protocols. More details are provided in the Supplemental Methods.

BRET assay. HEK293 cells were transiently transfected with WT and mutant (S54L) GFP-C $\beta$ (isoform 1) and the respective Renilla luciferase-tagged (RLuc-tagged) regulatory subunit using polyethyleneimine $(25 \mathrm{kDa}$, Polysciences $\mathrm{GmbH})$. The reporter proteins were expressed for 48 hours. For endpoint measurements, cells were washed with Dulbecco's phosphate-buffered saline (DPBS, Biowest) and incubated with $5 \mu \mathrm{M}$ (final concentration) Coelenterazine 400A (BIOTREND Chem.) supplemented either with $50 \mu \mathrm{M}$ forskolin (Sigma-Aldrich) and $100 \mu \mathrm{M}$ IBMX (Sigma-Aldrich) or DPBS alone (untreated control) for 20 minutes. Data acquisition was performed on a POLARstar Omega microplate reader (dual emission optics, BMG Labtech) using filters at wavelengths $410 \pm 80 \mathrm{~nm}$ (donor) and 515 $\pm 30 \mathrm{~nm}$ (acceptor). Emission values for nontransfected cells were subtracted for referencing. Control measurements with cells expressing RLuc8 alone were performed for each experiment. Mean values ( \pm SD) were calculated from 3 independent measurements (each from 3-6 replicates).

For kinetic experiments, transfected cells were washed with DPBS and the reaction was started by the addition of $5 \mu \mathrm{M}$ Coelenterazine 400A in DPBS. After 9 minutes, the cell-permeant precursor of 8-BrcAMP (8-Br-cAMP-AM, B020, Biolog) was injected at a final concentration of $100 \mu \mathrm{M}$ in DPBS supplemented with $5 \mu \mathrm{M}$ Coelenterazine $400 \mathrm{~A}$ using the reagent injector of the microplate reader.

Interaction analysis using SPR. A Biacore T200 SPR instrument (GE Healthcare) was used for kinetic analysis of the interaction of PKA-C $\beta$ WT and S54L with GST-PKA-RI $\alpha$. For this, an anti-GST (glutathione S-transferase) antibody (Carl Roth) functionalized CM5 sensor chip (GE Healthcare) was used as described previously (35). GST-PKA-RI $\alpha$ was captured on this anti-GST antibody surface and several concentrations of either WT (0-8 nM) or S54L (0-32 nM) PKA-C $\beta$ (isoform 1) were injected in running buffer (20 mM MOPS, pH 7, $150 \mathrm{mM} \mathrm{NaCl}, 50 \mu \mathrm{M}$ EDTA, $1 \mathrm{mM}$ ATP, $10 \mathrm{mM} \mathrm{MgCl}{ }_{2}, 0.01 \%$ P20) at a flow rate of $30 \mu \mathrm{l} / \mathrm{min}$ at $25^{\circ} \mathrm{C}$ for 300 seconds. Dissociation of the PKA-C-RI $\alpha$ complex was tested by switching 
to buffer without PKA-C for 300 seconds. Regeneration of the chip surface was performed by injecting 10 $\mathrm{mM}$ glycine ( $\mathrm{pH}$ 1.9) for 30 seconds at $30 \mu \mathrm{l} / \mathrm{min}$ at the end of each measurement cycle. Binding of PKA$\mathrm{C} \beta$ to GST-tagged PKI $\alpha$ was performed as described previously (35). Rate constants were determined by nonlinear curve fitting (global fit analysis) using the software T200 Evaluation 3.0 (GE Healthcare).

In order to test the cAMP sensitivity of WT and mutant PKA holoenzymes using SPR, GST-PKA-RI $\alpha$ was captured on the anti-GST antibody surface (30-40 response units [RU]) and $15 \mathrm{nM}$ PKA-C $\beta$ (WT or $\mathrm{S} 54 \mathrm{~L}$ ) was injected in running buffer for 150 seconds to monitor association of the holoenzyme. Dissociation (buffer without PKA-C) was monitored for 250 seconds. To analyze cAMP-induced dissociation, running buffer containing cAMP at several concentrations (ranging from $0 \mathrm{nM}$ to $320 \mathrm{nM}$ ) was injected for another 60 seconds. Surface regeneration was again performed by injecting $10 \mathrm{mM}$ glycine (pH 1.9) for 30 seconds at $30 \mu \mathrm{l} / \mathrm{min}$ at the end of each measurement cycle. Dissociation rate constants of the cAMPinduced dissociation were determined using the software BIAevaluation 4.1.1.

In vitro PKA activity assay of purified proteins. The expression and purification of recombinant PKA subunits are described in the Supplemental Methods. Specific activity in U/mg ( $\mu \mathrm{mol} / \mathrm{min}$ per $\mathrm{mg}$ ) of purified proteins, recombinantly expressed in E. coli, were determined using a coupled spectrophotometric assay with kemptide (LRRASLG) according to Cook et al. (36). The same assay was used to determine apparent activation constants of WT and mutant holoenzymes. For this, holoenzymes were preformed by mixing PKA-RI $\alpha$ and the PKA-C $\beta$ WT or S54L (isoform 1) in a molar ratio of 1:1.2 or $1: 1.3$, respectively. The sample was then transferred into a D-tube dialyzer tube (MWCO 6-8 kDa, Millipore) and dialyzed against buffer D (20 mM MOPS, pH 7, $150 \mathrm{mM} \mathrm{NaCl}, 0.1 \mathrm{mM}$ ATP, $1 \mathrm{mM} \mathrm{MgCl}{ }_{2}$, $2 \mathrm{mM} \beta$-mercaptoethanol) at $4^{\circ} \mathrm{C}$. In the kinase assay a final kinase concentration of either $10 \mathrm{nM}$ (WT) or $20 \mathrm{nM}$ (S54L) was used. Preformed holoenzymes were incubated with cAMP (0.1 nM to $100 \mu \mathrm{M})$ for 1 minute prior to measurement. All measurements were done at least in duplicate and with at least 2 independent holoenzyme preparations.

In vitro PKA activity assay with cell lysates. All experiments were performed 48 hours after transfection. Cells were washed twice with PBS at room temperature, scraped from the plate, and resuspended in $300 \mu 1$ lysis buffer ( 5 mM Tris-HCl, 2 mM EDTA, pH 7.4). The cells were lysed using an Ultra-turrax (IKA) for 20 seconds on ice. Then the samples were centrifuged at $50,000 \mathrm{~g}$ for 30 minutes at $4^{\circ} \mathrm{C}$ to remove membranes. To check for equal input of catalytic subunit for the PKA activity assay, expression of PKA subunits FLAG-C $\beta$, $\mathrm{RI} \alpha$, and RII $\beta$ in cell lysates was determined by Western blot with specific antibodies (anti-FLAG $[1: 4,000]$, F7425, Sigma-Aldrich; anti-PKA RI $\alpha$ [1:1,000], 610609, BD Transduction Laboratories; anti-PKA RII $\beta$ $[1: 1,000], 610625$, BD Transduction Laboratories). PKA catalytic activity was measured with or without the addition of cAMP with the PepTag nonradioactive cAMP-dependent protein kinase assay (V5340, Promega) using kemptide, according to the manufacturer's instructions. Images of the gels were acquired with a gel documentation system (Herolab) and analyzed using ImageJ software (http://rsbweb.nih.gov/ij).

Immunohistochemistry. Immunohistochemistry was performed on serial sections from paraffin-embedded adrenal adenoma (the C $\beta$-S54L mutated adenoma and 2 WT adenomas). The immunohistochemical procedure is described in details in Supplemental Methods.

Statistics. All data are presented as the mean $\pm \mathrm{SD}$ and $P$ values were obtained by unpaired $t$ test or ANOVA with Sidak's post hoc test for multiple comparisons carried out using GraphPad Prism 6.

\section{Author contributions}

SE, JB, and CAS initiated and oversaw the study. SE, MJK, KB, GA, MRR, SF, WLR, and DA conducted experiments and acquired data. LG examined the patients and collected blood samples. SE and MJK wrote and JB, FWH, DC, and CAS edited the manuscript. All authors interpreted the data and contributed to the final version of the manuscript.

\section{Acknowledgments}

J. Bertherat's lab is supported by the E-RARE program (ANR-13-RARE-008) and the PROMEX foundation. C.A. Stratakis's lab is funded by the intramural research program of the NICHD (Z01 HD008920). F.W. Herberg's lab is funded by BMBF e:Bio project (0316177 F), DFG (He 1818/10). D. Calebiro's lab is funded by IZKF Würzburg (grant B281). S. Espiard and G. Assié are supported by INSERM. We thank I. Witzel and M. Hansch for excellent technical assistance and D. Bertinetti and M. Kaufholz for helpful discussions. 
Address correspondence to: Jérôme Bertherat, Service des Maladies Endocriniennes et Métaboliques, Hôpital Cochin, 27 rue du Faubourg St. Jacques, 75014 Paris, France. Phone: 33.14.44.12.390; Email: jerome.bertherat@cch.aphp.fr.

1. Taylor SS, Zhang P, Steichen JM, Keshwani MM, Kornev AP. PKA: lessons learned after twenty years. Biochim Biophys Acta. 2013;1834(7):1271-1278.

2. Skålhegg BS, Taskén K. Specificity in the cAMP/PKA signaling pathway. Differential expression, regulation, and subcellular localization of subunits of PKA. Front Biosci. 1997;2:d331-d342.

3. Espiard S, Bertherat J. The genetics of adrenocortical tumors. Endocrinol Metab Clin North Am. 2015;44(2):311-334.

4. Beuschlein F, et al. Constitutive activation of PKA catalytic subunit in adrenal Cushing's syndrome. N Engl J Med. 2014;370(11):1019-1028.

5. Cao Y, et al. Activating hotspot L205R mutation in PRKACA and adrenal Cushing's syndrome. Science. 2014;344(6186):913-917.

6. Sato Y, et al. Recurrent somatic mutations underlie corticotropin-independent Cushing's syndrome. Science. 2014;344(6186):917-920.

7. Goh G, et al. Recurrent activating mutation in PRKACA in cortisol-producing adrenal tumors. Nat Genet. 2014;46(6):613-617.

8. Calebiro D, et al. PKA catalytic subunit mutations in adrenocortical Cushing's adenoma impair association with the regulatory subunit. Nat Commun. 2014;5:5680.

9. Ronchi CL, et al. Genetic landscape of sporadic unilateral adrenocortical adenomas without PRKACA p.Leu206Arg mutation J Clin Endocrinol Metab. 2016;101(9):3526-3538.

10. Prinz A, Diskar M, Erlbruch A, Herberg FW. Novel, isotype-specific sensors for protein kinase A subunit interaction based on bioluminescence resonance energy transfer (BRET). Cell Signal. 2006;18(10):1616-1625.

11. Aimes RT, Hemmer W, Taylor SS. Serine-53 at the tip of the glycine-rich loop of cAMP-dependent protein kinase: role in catalysis, P-site specificity, and interaction with inhibitors. Biochemistry. 2000;39(28):8325-8332.

12. Bossemeyer D. The glycine-rich sequence of protein kinases: a multifunctional element. Trends Biochem Sci. 1994;19(5):201-205.

13. Gamm DM, Baude EJ, Uhler MD. The major catalytic subunit isoforms of cAMP-dependent protein kinase have distinct biochemical properties in vitro and in vivo. J Biol Chem. 1996;271(26):15736-15742.

14. Huang Y, Roelink H, McKnight GS. Protein kinase A deficiency causes axially localized neural tube defects in mice. $J$ Biol Chem. 2002;277(22):19889-19896.

15. Qi M, et al. Impaired hippocampal plasticity in mice lacking the Cbeta1 catalytic subunit of cAMP-dependent protein kinase. Proc Natl Acad Sci USA. 1996;93(4):1571-1576.

16. Chen Y, Gao Y, Tian Y, Tian DL. PRKACB is downregulated in non-small cell lung cancer and exogenous PRKACB inhibits proliferation and invasion of LTEP-A2 cells. Oncol Lett. 2013;5(6):1803-1808.

17. Wu KJ, Mattioli M, Morse HC, Dalla-Favera R. c-MYC activates protein kinase A (PKA) by direct transcriptional activation of the PKA catalytic subunit beta (PKA-Cbeta) gene. Oncogene. 2002;21(51):7872-7882.

18. Ihara T, et al. An in vivo screening system to identify tumorigenic genes. Oncogene. 2017;36(14):2023-2029.

19. Padmanabhan A, Li X, Bieberich CJ. Protein kinase A regulates MYC protein through transcriptional and post-translational mechanisms in a catalytic subunit isoform-specific manner. J Biol Chem. 2013;288(20):14158-14169.

20. Shuntoh H, Sakamoto N, Matsuyama S, Saitoh M, Tanaka C. Molecular structure of the C beta catalytic subunit of rat cAMPdependent protein kinase and differential expression of $\mathrm{C}$ alpha and $\mathrm{C}$ beta isoforms in rat tissues and cultured cells. Biochim Biophys Acta. 1992;1131(2):175-180.

21. O'Hayre M, et al. The emerging mutational landscape of G proteins and G-protein-coupled receptors in cancer. Nat Rev Cancer. 2013;13(6):412-424

22. Calebiro D, Di Dalmazi G, Bathon K, Ronchi CL, Beuschlein F. cAMP signaling in cortisol-producing adrenal adenoma. Eur J Endocrinol. 2015;173(4):M99-106.

23. Lodish M, Stratakis CA. A genetic and molecular update on adrenocortical causes of Cushing syndrome. Nat Rev Endocrinol. 2016;12(5):255-262.

24. Thiel A, et al. PRKACA mutations in cortisol-producing adenomas and adrenal hyperplasia: a single-center study of 60 cases Eur J Endocrinol. 2015;172(6):677-685.

25. Di Dalmazi G, et al. Novel somatic mutations in the catalytic subunit of the protein kinase A as a cause of adrenal Cushing's syndrome: a European multicentric study. J Clin Endocrinol Metab. 2014;99(10):E2093-E2100.

26. Cancer Genome Atlas Research Network, et al. The Cancer Genome Atlas Pan-Cancer analysis project. Nat Genet. 2013;45(10):1113-1120.

27. Zheng S, et al. Comprehensive pan-genomic characterization of adrenocortical carcinoma. Cancer Cell. 2016;30(2):363.

28. Forlino A, et al. PRKACB and Carney complex. N Engl J Med. 2014;370(11):1065-1067.

29. Larkin SJ, et al. Sequence analysis of the catalytic subunit of PKA in somatotroph adenomas. Eur J Endocrinol. 2014;171(6):705-710.

30. Gicquel C, et al. Molecular markers and long-term recurrences in a large cohort of patients with sporadic adrenocortical tumors. Cancer Res. 2001;61(18):6762-6767.

31. Libé R, et al. Phosphodiesterase 11A (PDE11A) and genetic predisposition to adrenocortical tumors. Clin Cancer Res. 2008;14(12):4016-4024.

32. Schwarz JM, Cooper DN, Schuelke M, Seelow D. MutationTaster2: mutation prediction for the deep-sequencing age. Nat Methods. 2014;11(4):361-362.

33. Adzhubei IA, et al. A method and server for predicting damaging missense mutations. Nat Methods. 2010;7(4):248-249.

34. Ng PC, Henikoff S. SIFT: Predicting amino acid changes that affect protein function. Nucleic Acids Res. 2003;31(13):3812-3814.

35. Knape MJ, et al. Divalent metal ions $\mathrm{Mg}^{2+}$ and $\mathrm{Ca}^{2+}$ have distinct effects on protein kinase A activity and regulation. ACS Chem 
Biol. 2015;10(10):2303-2315.

36. Cook PF, Neville ME, Vrana KE, Hartl FT, Roskoski R. Adenosine cyclic 3',5'-monophosphate dependent protein kinase: kinetic mechanism for the bovine skeletal muscle catalytic subunit. Biochemistry. 1982;21(23):5794-5799. 